\title{
The Gender Gap in Science
}

\section{by Mei-Hung Chiu, Marie-Francoise \\ Roy, and \\ Hongming Liaw \\ n 2017, the ICSU \\ Gender-Gap in \\ Science project \\ was approved. Lead \\ by the International \\ Mathematical Union (IMU) and the \\ International Union of Pure and Applied Chemistry \\ (IUPAC), the project full title is "A Global Approach \\ to the Gender Gap in Mathematical and Natural \\ Sciences: How to Measure It, How to Reduce It?”}

Throughout history highly creative women have made their contributions to the world in mathematical and natural sciences. However, the percentages of women scientists remain shockingly low, and there is a significant gender gap at all levels between women and men. While barriers for women still exist, one of the major goals of the UN's Sustainable Development Goals, namely Goal 5: "Achieve gender equality and empower all women and girls," should receive more attention

Global Survey of Mathematical, Computing, and Natural Scientists: How to Measure It, How to Reduce It?

In 2010, the International Union of Pure and Applied Physics (IUPAP) and the American Institute of Physics (AIP) conducted the Global Survey of Physicists comparing experiences of men and women physicists around the world. There were 14,932 respondents from 130 countries and 8 languages. The results highlighted contrasts between women and men, and between less and more highly developed countries. This survey was valuable in understanding gender issues such as differential access to resources, opportunities for men and women on a country-by-country basis, and cultural expectations concerning child care. It aided decisions on where interventions are best targeted.

In this project, the global survey will be improved and extended to chemistry, astronomy, biology and mathematics. It will target 45,000 respondents (men and women) in multiple languages. In order to have a better knowledge of the gender gap in science around the world, the survey will explore comparisons across regions, countries, disciplines, level of development of the country (more developed or less developed), sector of employment (academia, industry, school), than it has in the past across the world. Therefore, we not only intend to focus our study in developed countries, but more importantly also in emerging countries, to empower human resources for societies.

The objectives of the project are to:

a. Provide evidence via both a joint global survey and a study of publication patterns to provide reliable data on which to orient future actions.

b. Collaborate with social scientists working in gender and science, obtaining contrasts and commonalities across regions and cultures, less and more highly developed countries, and across different disciplines.

c. Provide easy access to materials to encourage young women to work in our fields, including information about careers and salaries directed at parents, schools, and others who influence the careers of girls, in particular in the developing world.

d. Recommend practical policies and actions that will reduce the gender gap.

To achieve these objectives, three tasks were devised:

Task 1. Develop and conduct a new global survey

Task 2. Conduct a data-backed study on publication patterns

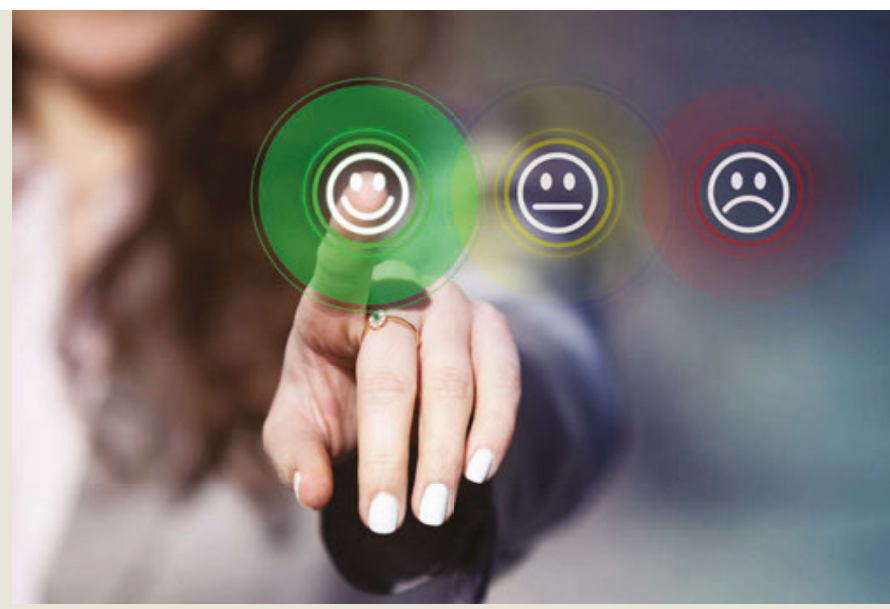

and age (younger and older respondents). It will also look at scientists' development of interest in science, experiences in education and careers, family support, access to resources needed to conduct science, and opportunities to contribute to the scientific enterprise. The survey will use special input from the three major regions of the ICSU Regional Offices-Africa, Asia and the Pacific, and Latin America and the Caribbean.

The 2018 Global Survey was launched on 1 May 2018 and will be opened for answers until 31 October 2018. Visit https://icsugendergapinscience.org for update. 
well as 12 local scholars and two local science teachers. The National Taiwan Normal University hosted the workshop, and the Chemical Society Located in Taipei also provided financial support. A report of the workshop can be found at https://icsugendergapinscience. org/2017/11/09/report-on-the-regional-workshopat-ntnu-taiwan/.

In the workshop 18 attendees from all over Asia and Australasia, representing various supporting organizations and disciplines, gave brief presentations on the status and/or issues of gender gap in science in their respective fields or countries. Presentations given by the attendees were as follows: An overall project overview was delivered by Prof. Marie-Francoise Roy from IMU and Prof. Mei-Hung Chiu from IUPAC as the lead Union 1 and 2 respectively. From IMU, Prof. Nalini Joshi (Australia) presented "Reflections on Gender Diversity in Mathematics;" Prof. Neela Nataraj (India) provided "A Global Approach to the Gender Gap in Mathematical and Natural Sciences: How to Measure It, How to Reduce It?"; and Prof. Dhana Thapa (Nepal) presented "Mathematics and Nepalese Women: A Glimpse." From IUPAC, Prof. Chiu (Taiwan) introduced the structure and mission of IUPAC as well as the Distinguished Women in Chemistry or Chemical Engineering IUPAC Awards. Prof. Rachel Mamlok-Naaman (Israel) gave an introduction to IUPAC and an overview on women's participation in sciences in Israel. Meanwhile, Dr. Supriya Saha (India) provided some "Best Practices for Promoting Female Scientists in India."

Hailing from ICIAM, Prof. Amy Novick-Cohen (Israel) overviewed "Gender Gap in Applied Math, Math and Materials, in Israel and Abroad." Dr. Kazue Sako (Japan) informed us on the JSIAM activities that were designed to encourage women researchers, and Prof. Guiying Yan (China) gave us a glimpse into the state of Chinese female scientific research.

For IUPAP, Prof. Youngah Park (Korea) provided some ideas on "How to Promote Gendered Innovations in Global S\&T Landscapes." Prof. Prajval Shastri (India) discussed "The Gender Gap in Science: Do the Causes Lie Within?"; and Prof. Ling-An Wu (China) gave an introduction on "Lecture Tours by Women Physicists in China".

In addition, Prof. Saeko Hayashi (IAU; Japan) talked about the "Mighty Minority of Women in Astronomy in Japan." Dr. Parawee Lekprasert and Dr. Chalita Thanyakoop (SAGA; Thailand) described "STEM and Gender Equality in Thailand." Prof. Tien Huynh (IUBS; Australia) gave a personal account of "My Journey in STEMM." Prof. Amita Chatterjee (IHUPST; India) presented "Gender Gap in Mathematics and Science: Some Observations from the Indian Perspective." Prof. Catherine Lang (ACM; Australia) introduced the Association for Computing Machinery - Women in Computing (ACM-W) regarding its highlights, objectives, and activities.

Among the local scholars, Prof. Chia-Li Wu (Taiwan) gave a presentation on the surveys of gender gap in Asia, and Prof. I-Ling Chang (Taiwan) described gender gap in science and engineering schools in Taiwan.

The above presentations were divided into three sessions. The first session was presided by Dr. Shihoko Ishii (IMU; Japan), the second

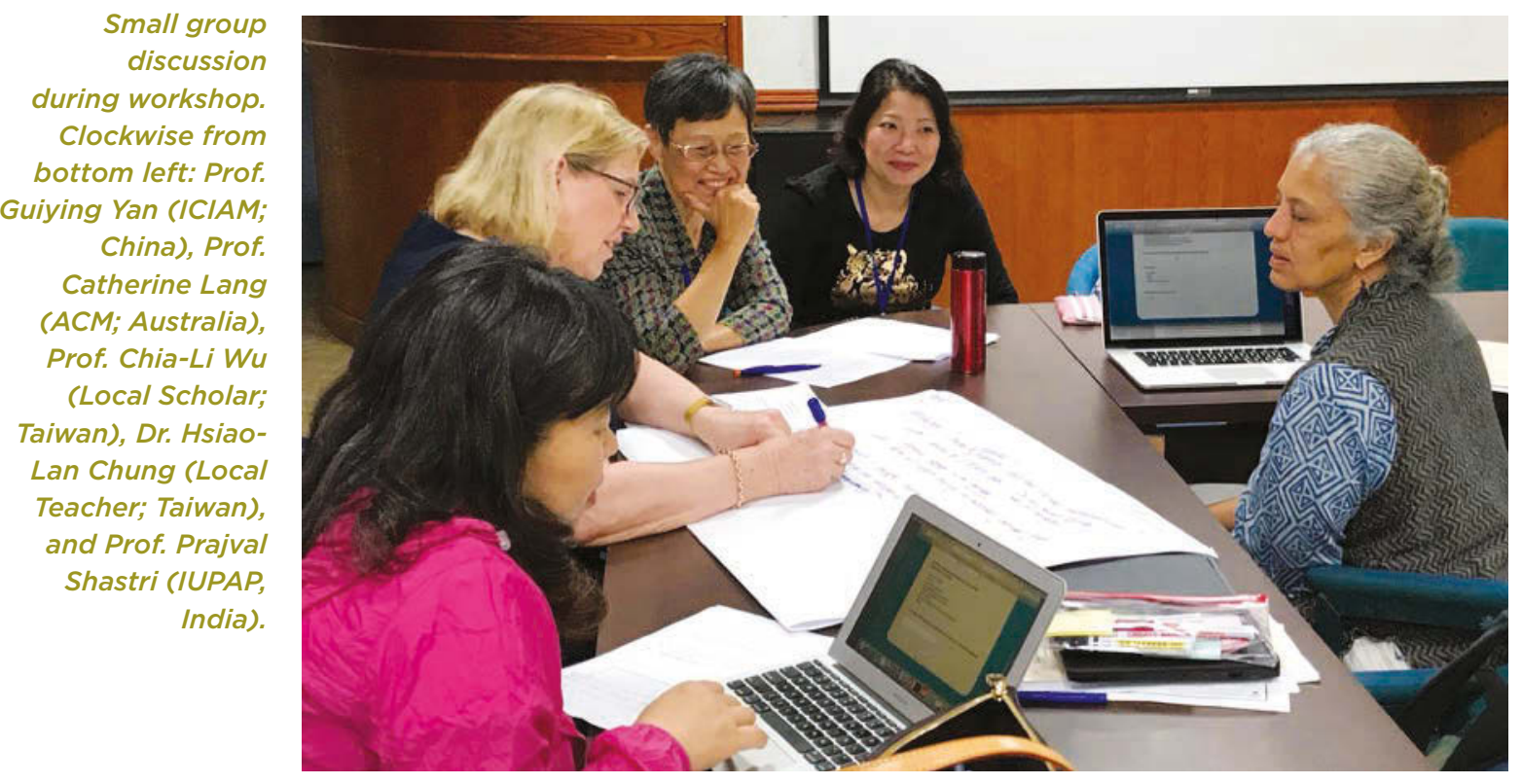


by Dr. Noriko Osumi (IUBS; Japan), and the last session was presided by Dr. Ting-Kueh Soon (IUPAC; Malaysia). Other attendees were from local universities, academia sinica, and high schools.

These brief presentations were then followed by Dr. Rachel Ivie of American Institute of Physics (AIP), who is responsible for the design of the global questionnaire.

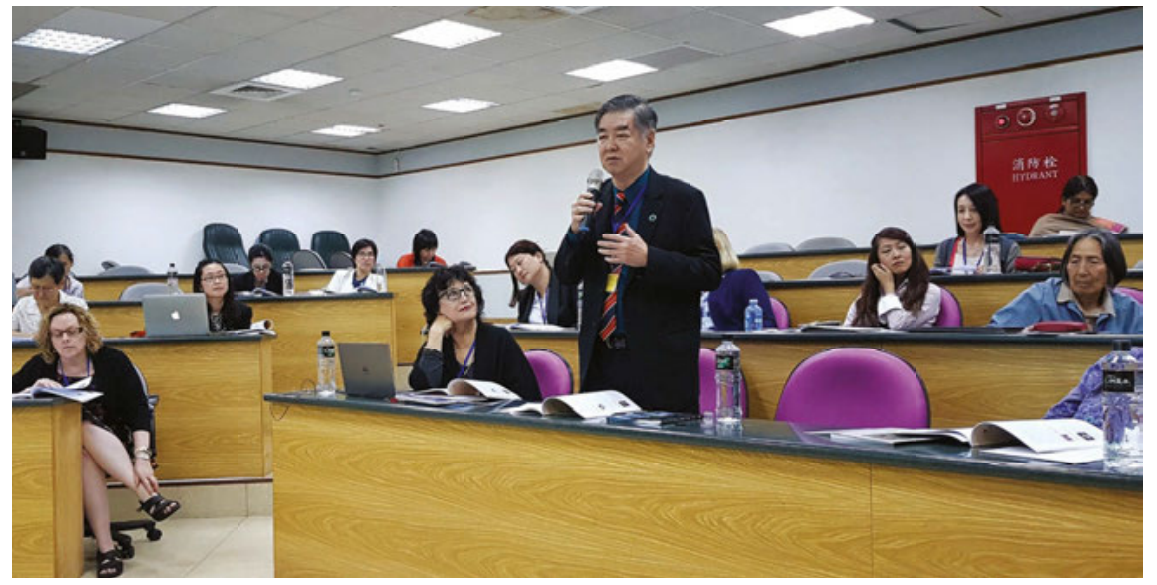

Dr. Ivie held several sessions

in the two-day workshop to understand attendees' concerns and suggestions for the questionnaire as well as the study of publication patterns in physics, chemistry, astronomy, biology, and mathematics. During her session, in addition to introducing the questionnaire to the participants, Dr. Ivie also held grouped activities during which participants can give more detailed and specific inputs to the questionnaire after reaching group-based consensus. The activities were greatly successful with ample useful inputs from all groups.

Overall feedback from attendees proved positive. One attendee stated "The workshop was excellent, informative, well-structured and very well-organized." Other attendees described the workshop as "warm and constructive," "a very effective and productive workshop," and having given "a new perspective of other disciplines in science." Other quantitative measures also revealed an overwhelmingly positive feedback among our attendees, with most attendees confirming that they felt the program of the workshop to be well-organized (average 4.88/5). Respondents of the post-workshop survey strongly felt that they have learned from the workshop: gender gap research across various countries (average 4.8/5) and issues across different disciplines (average 4.56/5). Through this workshop, respondents stated that they have shared their ideas for the future work for the project (average 4.44/5) and believed that they have made contributions to the draft questionnaire (average 4.56/5); they think that the results of the questionnaire will bring concrete recommendation for policies (average 4.56/5). By bringing so many attendees from across Asia and Australasia, respondents also believed that they have made new connections with participants through the workshop (average 4.72/5).
During the Q\&A session of the workshop, Datuk Dr Soon Ting Kueh shared his perspective of the challenges encountered in Malaysia.

The Taipei workshop was the first of the workshops and had provided valuable and essential inputs from scientists and mathematicians of Asia and Australasia.

Two additional workshops were later held in South America and Africa soon after the conclusion of the Taipei workshop: The Latin American Regional Workshop-the second workshop-took place at Universidad de los Andes in Bogotá, Colombia, on 22-24 November 2017 and was attended by 30 participants from 10 countries (Argentina, Brazil, Chile, Colombia, Costa Rica, Cuba, El Salvador, Mexico, Perú, USA). The third regional workshop took place at the African Institute of Mathematical Sciences, AIMS, Muizenberg, Cape Town, South Africa, on 1-2 December 2017.

With additional inputs from scholars and science practitioners from these two regions, it is hoped that the questionnaire will not only be more representative but also address cultural nuances and regional concerns, making the finalized questionnaire a truly global instrument.

The official survey was launched on 1 May 2018 and will last until 31 October 2018. Please help to disseminate the link and fill out the questionnaire, and help the science community to measure and reduce the gender gap. is

Mei-Hung Chiu and Hongming Liaw represent National Taiwan Normal University and IUPAC, Marie-Francoise Roy represents the Institute of Mathematical Union, and is a member of the Committee for Women in Mathematics

https://icsugendergapinscience.org 